\title{
3 \\ . \\ Survey around the Impacts of Cloud Computing in Mobile Environments
}

\author{
Sam Mathews M \\ Department Of Information Technology, Satyabama University, Chennai \\ Email mathews.sham@gmail.com
}

\begin{abstract}
Mobile Cloud Computing (MCC) which combines mobile computing and cloud computing, has become one of the industry buzz words and a major discussion thread in the IT world since 2009 . Despite increasing usage of mobile computing, exploiting its full potential is difficult due to its inherent problems such as resource scarcity, frequent disconnections, and mobility. MCC integrates the cloud computing into the mobile environment and overcomes obstacles related to the performance (e.g., battery life,storage, and bandwidth), environment (e.g., heterogeneity, scalability, and availability), and security (e.g., reliability and privacy) discussed in mobile computing. As MCC is still at the early stage of development, it is necessary to grasp a thorough understanding of the technology in order to point out the direction of future research. With the latter aim, this paper presents a review on the background and principle of MCC, characteristics, recent research work, and future research trends.
\end{abstract}

\section{Keywords: Cloud computing, Distributed Computing, Mobile Cloud Computing}

\section{INTRODUCTION}

Mobile devices (e.g., smartphone, tablet pcs, etc) are increasingly becoming an essential part of human life as the most effective and convenient communication tools not bounded by time and place. Mobile users accumulate rich experience of various services from mobile applications (e.g., iPhone apps, Google apps, etc), which run on the devices and/or on remote servers via wireless networks. The rapid progress of mobile computing (MC) ) [1] becomes a powerful trend in the development of IT technology as well as commerce and industry fields. However, the mobile devices are facing many challenges in their resources (e.g., battery life, storage, and bandwidth) and communications (e.g., mobility and security) [2]. The limited resources significantly impede the improvement of service qualities. Cloud computing (CC) has been widely recognized as the next generation's computing infrastructure. $\mathrm{CC}$ offers some advantages by allowing users to use infrastructure (e.g., servers, networks, and storages), platforms (e.g., middleware services and operating systems), and softwares (e.g., application programs).In short, we are rapidly approaching a time when computing will be:

- $\quad$ itinerant, maintaining full connectivity and computational power while moving with a person, in a vehicle, or on an aircraft or ship;

- distributed, integrating functions that are performed at different places in a way that is transparent to the user; and

- ubiquitous, providing the same functionality independent of the user's location

Of course we are still some way from achieving this ideal, and it is still easier and cheaper to compute in the conventional manner at a desktop using only the data, software, and hardware present in the workstation. Wireless communication is still more expensive, and more constrained by bandwidth, at least in those areas of the developed world that are well-served by copper and fiber. But the pace of change is rapid, and in some countries we are already approaching the point where the majority of telephone communication will be wireless. New devices and methods of communication suggest the need for a fundamental rethinking of the principles of geographic information systems design. Computers are likely to be used in new kinds of places that are very different from the conventional office; to communicate information using new modes of interaction, such as the pen; and to require much better use of restricted displays and computing power. On the other hand, devices are already available that offer the power of a desktop machine in a wearable package. All of these developments challenge the research community to investigate entirely new applications, and to address technical problems. Delivering cloud services in a mobile environment brings numerous challenges and problems. Mobile devices cannot handle complicated applications due to their innate characters. Also, it is impossible that a mobile device is always online, the offline solution of the device need be 
considered as well. The absence of standards, security and privacy, elastic mobile applications requirement may obstruct the development of Mobile Cloud Computing. In order to understand the challenges and provide further scope for research, an understanding of this novel approach is essential. This paper introduces the basic model of MCC, its background, key technology, challenges, current research status and future research perspectives. The paper is organized as follows: Section I gives an introduction to the technology, Section II gives the background of mobile and cloud is presented, Section III gives an overview of mobile cloud, Section IV presents Applications, Section $\mathrm{V}$ presents Open Research Issues. The conclusions are drawn in Section

\section{BACKGROUND}

As a development and extension of Cloud Computing and Mobile Computing, Mobile Cloud Computing, as a new phrase, has been devised since 2009. In order to help us grasping better understanding of Mobile Cloud Computing, let's start from the two previous techniques: Mobile Computing and Cloud Computing.

\section{A. Mobile Computing:}

Mobility has become a very popular word and rapidly increasing part in today's computing area. An incredible growth has appeared in the development of mobile devices such as, smartphone, PDA, GPS Navigation and laptops with a variety of mobile computing, networking and security technologies. In addition, with the development of wireless technology like WiMax, Ad Hoc Network and WIFI, users may be surfing the Internet much easier but not limited by the cables as before. Thus, those mobile devices have been accepted by more and more people as their first choice of working and entertainment in their daily lives.

So, what is Mobile computing exactly? In Wikipedia, it is described as a form of human-computer interaction by which a computer is expected to be transported during normal usage [8]. Mobile computing is based on a collection of three major concepts: hardware, software and communication. The concepts of hardware can be considered as mobile devices, such as smartphone and laptop, or their mobile components. Software of mobile computing is the numerous mobile applications in the devices, such as the mobile browser, anti-virus software and games. The communication issue includes the infrastructure of mobile networks, protocols and data delivery in their use.

They must be transparent to end users.

Features: the features of mobile computing are as follows:

a) Mobility: mobile nodes in mobile computing network can establish connection with others, even fixed nodes in wired network through Mobile Support Station (MSS) during their moving. b) Diversity of network conditions: normally the net works using by mobile nodes are not unique, such networks can be a wired network with highbandwidth, or a wireless Wide Area Network (WWAN) with low-bandwidth, or even in status of disconnected.

c) Frequent disconnection and consistency: as the limitation of battery power, charge of wireless communication,

network conditions and so on, mobile nodes will not always keep the connection, but disconnect and consistent with the wireless network passively or actively.

d) Dis-symmetrical network communication: servers and access points and other MSS enable a strong send/receive ability, while such ability in mobile nodes is quite weak comparatively. Thus, the communication bandwidth and overhead between downlink and uplink are discrepancy.

e) Low reliability: due to signals is susceptible to inter-ference and snooping, a mobile computing network system has to be considered from terminals, networks, database platforms, as well as applications development to address the security issue.

Challenges: Compared with the traditional wired network, mobile computing network may face various problems and challenges in different aspects, such as signal disturbance, security, hand-off delay, limited power, low computing ability, and so on. due to the wireless environment and numerous mobile nodes. In addition, the Quality of Service (QoS) in mobile computing network is much easier to be affected by the landforms, weather and buildings.

\section{B. Cloud Computing:}

In the era of PC, many users found that the PCs they bought 2 years ago cannot keep pace with the development of software nowadays; they need a higher speed CPU, a larger capacity hard disk, and a higher performance Operation System (OS). That is the magic of 'Moores Law' which urges user upgrading their PCs constantly, but never ever overtaken the development of techniques. Thus, a term called 'Cloud Computing' burst upon our lives.

Cloud Computing has become a popular phrase since 2007. However, there is no consensual definition on what a Cloud Computing or Cloud Computing System is, due to dozens of developers and organizations described it from different perspectives. C. Hewitt [9] introduces that the major function of a cloud computing system is storing data on the cloud servers, and uses of cache memory technology in the client to fetch the data. Those clients can be PCs, laptops, smartphones and so on. R. Buyya [10] gives a definition from the perspective of marking that cloud computing is a parallel and distributed computing system, which is 
combined by a group of virtual machines with internal links. Such systems dynamically offer computing resources from service providers to customers according to their Service level Agreement (SLA). However, some authors mentioned that cloud computing was not a completely new concept. L. Youseff [11] from UCSB argue that cloud computing is just combined by many existent and few new concepts in many research fields, such as distributed and grid computing, ServiceOriented Architectures (SOA) and in virtualization.

In this paper, we consider the cloud computing is a large -scale economic and business computing paradigm with virtualization as its core technology. The cloud computing system is the development of parallel processing, distributed and grid computing on the Internet, which provides various QoS guaranteed services such as hardware, infrastructure, platform, software and storage to different Internet applications and users.

Framework: cloud computing systems actually can be considered as a collection of different services, thus the framework of cloud computing is divided into three layers, which are infrastructure layer, platform layer, and application layer (see Fig 1)

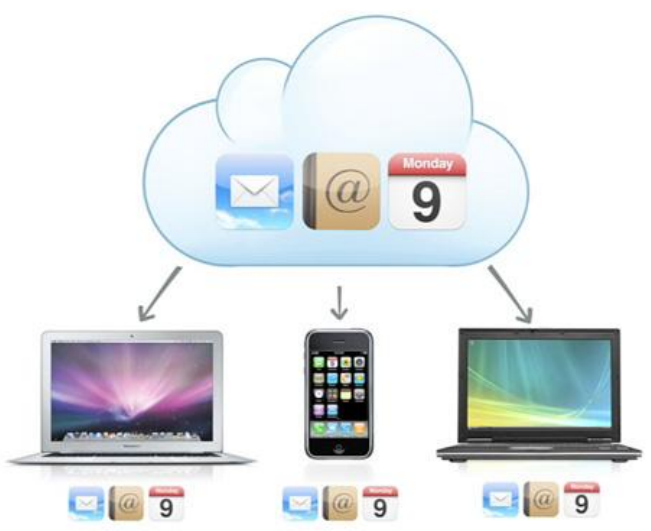

Fig 1 Mobile- Cloud Environment

a) Infrastructure layer: it includes resources of com-puting and storage. In the bottom layer of the framework, physical devices and hardware, such as servers and storages are virtualized as a resource pool to provide computing storage and network services users, in order to install operation system(OS) and operate software application. Thus it is denoted as Infrastructure as a Service (IaaS). Typically services in this layer such as Elastic Computing Cloud of Amazon [12].

b) Platform layer: this layer is considered as a core layer in the cloud computing system, which includes the environ ment of parallel programming design, distributed storage andmanagement system for structured mass data, distributed file system for mass data, and other system management tools for cloud computing. Program developers are the major clients of the platform layer. All platform resources such as program testing, running and maintaining are provided by the platform directly but not to end users. Thus, this type of services in a platform layer is called Platform as a Service (PaaS). The typical services are Google App Engine [15] and Azure from Microsoft [14].

c) Application layer: this layer provides some simple software and applications, as well as costumer interfaces to endusers. Thus we name this type of services in the application layer as Software as a Service (SaaS). Users use client software or a browser to call services from providers through the Internet, and pay costs according to the utility business model (like water or electricity) [15]. The earliest SaaS is the Customer Relationship Management (CRM) [16] from Salesforce, which was developed based on the force.com (a PaaS in Salesforce). Some other services provided by Google on-line office such as documents, spreadsheets, presentations are all SaaS.

Features: the features of Cloud Computing are as follows:

a) Virtualization: the 'Cloud' can be considered as a virtual resource pool [16] where all bottom layer hardware devices is virtualized. End users access desired resources through a browser and get data from cloud computing providers without maintaining their own data centres. Furthermore, some virtual machines (VMs) are often installed in a server in order to improve the efficiency to use resources; and such VMs support load migration when there is a server over-load.

b) Reliability, usability and extensibility: cloud comput-ing provides a safe mode to store user's data while users do not worry about the issues such as software updating, leak patching, virus attacks and data loss. If failure happens on a server or VM, the cloud computing systems transfer and backup those data to other machines, and then delete those failure nodes from the systems automatically in order to make sure the whole system has normal operation [17]. Meanwhile, cloud can be extended from horizontal and vertical [18] in a large-scale network, to process numerous requests from thousands of nodes and hosts.

c) Large-scale: in order to possess the capability of supercomputing and mass storage, a cloud computing system normally consists of thousands of servers and PCs. Google Cloud Computing, for example, has already controlled $2 \%$ of all servers or about 1 million servers located in two hundred different places in the world, and will move upward to 10 million servers in the next decade [19].

d) Autonomy: a cloud system is an autonomic 
system, which automatically configures and allocates the resources of hardware, software and storage to clients on-demand, and the management is transparent to end users.

Challenges: First of all, cloud computing needs an improved mechanism to provide a safe and high efficiency service as the numerous invoked third-party software and infrastructures are implementing in computing. In addition, due to data centres of resource using a mass of electricity, efficient resource scheduling strategy and methods are required in order to save energy. Furthermore, as a Service Level Agreement (SLA) is established between users and service providers in cloud computing, so the performance and analysis of services are necessary to be monitored. Last but not least, simple and convenient application interfaces are indispensable for service providers in cloud computing, thus a uniform standard is required eagerly.

\section{OVERVIEW OF MOBILE CLOUD COMPUTING}

The term "mobile cloud computing" was introduced not long after the concept of "cloud computing" launched in mid-2007. It has been attracting the attentions of entrepreneurs as a profitable business option that reduces the development and running cost of mobile applications, of mobile users as a new technology to achieve rich experience of a variety of mobile services at low cost, and of researchers as a promising solution for green IT [20]. This

section provides an overview of MCC including definition, architecture, and advantages of MCC.

\section{A. Mobile Cloud Computing:}

The Mobile Cloud Computing Forum defines MCC as follows [21]: "Mobile Cloud Computing at its simplest, refers to an infrastructure where both the data storage and the data processing happen outside of the mobile device. Mobile cloud applications move the computing power and data storage away from mobile phones and into the cloud, bringing applications and mobile computing to not just smartphone users but a much broader range of mobile subscribers".

Aepona [22] describes MCC as a new paradigm for mobile applications whereby the data processing and storage are moved from the mobile device to powerful and centralized computing platforms located in clouds. These centralized applications are then accessed over the wireless connection based on a thin native client or web browser on the mobile devices. Alternatively, MCC can be defined as a combination of mobile web and cloud computing [23], [24], which is the most popular tool for mobile users to access applications and services on the Internet. Briefly, MCC provides mobile users with the data processing and storage services in clouds. The mobile devices do not need a powerful configuration (e.g., CPU speed and memory capacity) since all the complicated computing modules can be processed in the clouds.

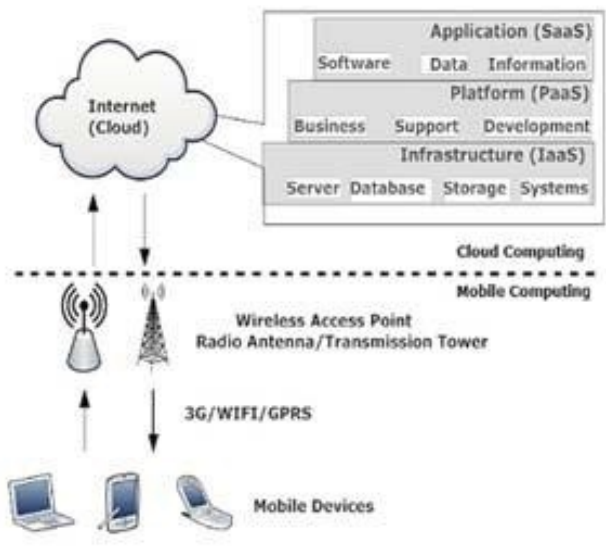

Fig 2. Mobile Cloud Computing (MCC) Architecture.

From the concept of MCC, the general architecture of MCC can be shown in Fig. 2. In Fig. 2, mobile devices are connected to the mobile networks via base stations (e.g., base transceiver station (BTS), access point, or satellite) that establish and control the connections (air links) and functional interfaces between the networks and mobile devices. Mobile users' requests and information (e.g., ID and location) are transmitted to the central processors that are connected to servers providing mobile network services. Here, mobile network operators can provide services to mobile users as AAA (for authentication, authorization, and accounting) based on the home agent (HA) and subscribers' data stored in databases. After that, the subscribers' requests are delivered to a cloud through the Internet. In the cloud, cloud controllers process the requests to provide mobile users with the corresponding cloud services. These services are developed with the concepts of utility computing, virtualization, and service-oriented architecture (e.g., web, application, and database servers).

Nowadays, both hardware and software of mobile devices get greater improvement than before, some smartphones such as iPhone 4S, Android serials, Windows Mobile serials and Blackberry, are no longer just traditional mobile phones with conversation, SMS, Email and website browser, but are daily necessities to users. Meanwhile, those smartphones include various sensing modules like navigation, optics, gravity, orientation, and so on. which brings a convenient and intelligent mobile experience to users. In 2010, Google CEO Eric Schmidt described mobile cloud computing in an interview that 'based on cloud computing service development, mobile phones will become increasingly complicated, and evolve to a portable super computer' [25]. In the face of various mobile cloud services provided by Microsoft, Apple, Google, HTC, and so on, users may be confused about what mobile cloud computing exactly is, and what its features are.

\section{B. Challenges and Solutions:}


The main objective of mobile cloud computing is to provide a convenient and rapid method for users to access and receive data from the cloud, such convenient and rapid method means accessing cloud computing resources effectively by using mobile devices. The major challenge of mobile cloud computing comes from the characters of mobile devices and wireless networks, as well as their own restriction and limitation, and such challenge makes application designing, programming and deploying on mobile and distributed devices more complicated than on the fixed cloud devices [26]. In mobile cloud computing environment, the limitations of mobile devices, quality of wireless communication, types of application, and support from cloud computing to mobile are all important factors that affect assessing from cloud computing. Table 1 gives an overview of proposed challenges and some solutions about mobile cloud computing.

Limitations of mobile devices: While discussing mobile devices in cloud the first thing is resourceconstrain. Though smartphones have been improved obviously in various aspects such as capability of CPU and memory, storage, size of screen, wireless communication, sensing technology, and operation systems, still have serious limitations such as limited computing capability and energy resource, to deploy complicated applications. By contrast with PCs and Laptops in a given condition, these smartphones like iPhone 4S, Android serials, Windows Mobile serials decrease 3 times in processing capacity, 8 times in memory, 5 to 10 times in storage capacity and 10 times in network bandwidth. Normally, smartphone needs to be charged everyday as dialling calls, sending messages, surfing the Internet, community accessing, and other internet applications. According to past development trends, the increased mobile computing ability and rapid development of screen technology will lead to more and more complicated applications deployed in smartphones. If the battery technology cannot be improved in a short time, then how to effectively save battery power in smartphone is a major issue we meet today.

Table 1: Challenges And Solutions Of Mobile Cloud Computing

\begin{tabular}{|l|l|}
\hline \multicolumn{1}{|c|}{ Challenges } & \multicolumn{1}{c|}{ Solutions } \\
\hline $\begin{array}{l}\text { Limitations of } \\
\text { mobile devices }\end{array}$ & $\begin{array}{l}\text { Virtualization and Image, } \\
\text { Task migration }\end{array}$ \\
\hline $\begin{array}{l}\text { Quality of } \\
\text { communication }\end{array}$ & $\begin{array}{l}\text { Bandwidth upgrading, Data } \\
\text { delivery time reducing }\end{array}$ \\
\hline $\begin{array}{l}\text { Division of } \\
\text { applications services }\end{array}$ & $\begin{array}{l}\text { Elastic application division } \\
\text { mechanism }\end{array}$ \\
\hline
\end{tabular}

The processing capacity, storage, battery time, and communication of those smartphones will be improved consistently with the development of mobile computing. However, such enormous variations will persist as one of major challenges in mobile cloud computing.

Quality of communication: In contrast with wired net work uses physical connection to ensure bandwidth consistency, the data transfer rate in mobile cloud computing environment is constantly changing and the connection is discontinuous due to the existing clearance in network overlay. Furthermore, data centre in large enterprise and resource in Internet service provider normally is far away to end users, especially to mobile device users. In wireless network, the network latency delay may $200 \mathrm{~ms}$ in 'last mile' but only $50 \mathrm{~ms}$ in traditional wired network. Some other issues such as dynamic changing of application throughput, mobility of users, and even weather will lead to changes in bandwidth and network overlay. Therefore, the handover delay in mobile network is higher than in wired network

Division of application services: In mobile cloud computing environment, due to the issue of limited resources, some applications of compute-intensive and data-intensive cannot be deployed in mobile devices, or they may consume massive energy resources. Therefore, we have to divide the applications and use the capacity of cloud computing to achieve those purposes, which is: the core computing task is processed by cloud, and those mobile devices are responsible for some simple tasks only. In this processing, the major issues affecting performance of mobile cloud computing are: data processing in data centre and mobile device, network handover delay, and data delivery time.

For a given standard, providing a quality guaranteed cloud service should consider the following facts: optimal division of application between cloud and mobile device, interaction between low-latency and code offload, high-bandwidth between cloud and mobile device for high speed data transmission, user-oriented cloud application performance, self-adaptation mechanism of mobile cloud computing, and optimal consumption and overhead of mobile devices and cloud servers. The following strategies can be used to response to the challenges:

- Upgrade bandwidth for wireless connection, make the web content more suitable for mobile network using regional data centres.

- Deploy the application processing node at the 'edge' of cloud in order to reduce data delivery time.

- Duplicate mobile devices to cloud using virtualization and image technologies, to process Data-Intensive Computing (DIC) and EnergyIntensive Computing, such as virus scanning in mobile devices.

- Dynamically optimize application push in cloud and the division with mobile terminals.

\section{APPLICATIONS OF MOBILE CLOUD COMPUTING}

Mobile applications gain increasing share in a global mobile market. Various mobile applications have taken the advantages of MCC. In this section, some typical MCC applications are introduced. A. Mobile Commerce 
Mobile commerce (m-commerce) is a business model for commerce using mobile devices. The mcommerce applications generally fulfill some tasks that require mobility (e.g., mobile transactions and payments, mobile messaging, and mobile ticketing). The m-commerce applications can be classified into a few classes including finance, advertising and shopping

\section{A. M-Commerce:}

The m-commerce applications have to face various challenges (e.g., low network bandwidth, high complexity of mobile device configurations, and security). Therefore, m-commerce applications are integrated into cloud computing environment to address these issues. [27] proposes a $3 \mathrm{G}$ E-commerce platform based on cloud computing. This paradigm combines the advantages of both $3 \mathrm{G}$ network and cloud computing to increase data processing speed and security level [28] based on PKI (public key infrastructure). The PKI mechanism uses an encryption-based access control and an over-encryption to ensure privacy of user's access to the outsourced data. In [29], a 4PL-AVE trading platform utilizes cloud computing technology to enhance the security for users and improve the customer satisfaction, customer intimacy, and cost competitiveness.

\section{B. Mobile Learning:}

Mobile learning (m-learning) is designed based on electronic learning (e-learning) and mobility. However, traditional m-learning applications have limitations in terms of high cost of devices and network, low network transmission rate, and limited educational resources [30], [31], [32]. Cloud-based m-learning applications are introduced to solve these limitations. For example, utilizing a cloud with the large storage capacity and powerful processing ability, the applications provide learners with much richer services in terms of data (information) size, faster processing speed, and longer battery life. [33] presents benefits of combining $\mathrm{m}$ learning and cloud computing to enhance the communication quality between students and teachers. In this case, a smartphone software based on the open source JavaME UI framework and Jaber for clients is used. Through a web site built on Google Apps Engine, students communicate with their teachers at anytime. Also, the teachers can obtain the information about student's knowledge level of the course and can answer students' questions in a timely manner. In addition, a contextual m-learning system based on IMERA platform [34] shows that a cloud-based m-learning system helps learners access learning resources remotely. Another example of MCC applications in learning is "Cornucopia" implemented for researches of undergraduate genetics students and "Plantations Pathfinder" designed to supply information and provide a collaboration space for visitors when they visit the gardens [35]. The purpose of the deployment of these applications is to help the students enhance their understanding about the appropriate design of mobile cloud computing in supporting field experiences. In [36], an education tool is developed based on cloud computing to create a course about image/video processing. Through mobile phones, learners can understand and compare different algorithms used in mobile applications (e.g., de-blurring, de-noising, face detection, and image enhancement).

\section{Mobile Healthcare:}

The purpose of applying MCC in medical applications is to minimize the limitations of traditional medical treatment (e.g., small physical storage, security and privacy, and medical errors [37], [38]). Mobile healthcare (m-healthcare) provides mobile users with convenient helps to access resources (e.g., patient health records) easily and quickly. Besides, $\mathrm{m}$ - healthcare offers hospitals and healthcare organizations a variety of on-demand services on clouds rather than owning standalone applications on local servers. There are a few schemes of MCC applications in healthcare. For example, [39] presents five main mobile healthcare applications in the pervasive environment. Comprehensive health monitoring services enable patients to be monitored at anytime and anywhere through broadband wireless communications. Intelligent emergency management system can manage and coordinate the fleet of emergency vehicles effectively and in time when receiving calls from accidents or incidents. Health-aware mobile devices detect pulse -rate, blood pressure, and level of alcohol to alert healthcare emergency system.

Pervasive access to healthcare information allows patients or healthcare providers to access the current and past medical information. Pervasive lifestyle incentive management can be used to pay healthcare expenses and manage other related charges automatically.

Similarly, [40] proposes@HealthCloud, a prototype implementation of m-healthcare information management system based on cloud computing and a mobile client running Android operating system (OS).This prototype presents three services utilizing the Amazon's S3 Cloud Storage Service to manage patient health records and medical images.Seamless connection to cloud storage allows users to retrieve, modify, and upload medical contents (e.g., medical images, patient health records and biosignals) utilizing web services and a set of available APIs called REST. Patient health record management system displays the information regarding patients' status, related biosignals and image contents through application's interface.Image viewing support allows the mobile users to decode the large image files at different resolution levels given different network availability and quality.

For practical system, a telemedicine homecare management system [41] is implemented in Taiwan to monitor participants, especially for patients with hypertension and diabetes. The system monitors 300 participants and stores more than 4736 records of blood pressure and sugar measurement data on the cloud. 
When a participant performs blood glucose/pressure measurement via specialized equipment, the equipment can send the measured parameters to the system automatically, or the participant can send parameters by SMS via their mobile devices. After that, the cloud will gather and analyze the information about the participant and return results. The development of mobile healthcare clearly provides tremendous helps for the participants. However, the information to be collected and managed related to personal health is sensitive. Therefore, [42], [43] propose solutions to protect participant's health information, thereby increasing the privacy of the services. While [44] uses P2P paradigm to federate clouds to address security issue, data protection and ownership, the model in provides security as a service on the cloud to protect mobile applications. Therefore, mobile health application providers and users will not have to worry about security issue since it is ensured by security vendor.

\section{Mobile Gaming:}

Mobile game ( $\mathrm{m}$ - game) is a potential market generating revenues for service providers. M-game can completely offload game engine requiring large computing resource (e.g., graphic rendering) to the server in the cloud, and gamers only interact with the screen interface on their devices. [44] demonstrates that offloading (multimedia code) can save energy for mobile devices, thereby increasing game playing time on mobile devices. [44] proposes MAUI (memory arithmetic unit and interface), a system that enables fine- grained energy-aware offloading of mobile codes to a cloud. Also, a number of experiments are conducted to evaluate the energy used for game applications with $3 \mathrm{G}$ network and WiFi network. It is found that instead of offloading all codes to the cloud for processing, MAUI partitions the application codes at a runtime based on the costs of network communication and CPU on the mobile device to maximize energy savings given network connectivity. The results demonstrate that MAUI not only helps energy reduction significantly for mobile devices (i.e., MAUI saves $27 \%$ of energy usage for the video game and $45 \%$ for chess), but also improves the performance of mobile applications (i.e., the game's refresh rate increases from 6 to 13 frames per second). [45] presents a new cloud-based m-game using a rendering adaptation technique to dynamically adjust the game rendering parameters according to communication constraints and gamers' demands. The rendering adaptation technique mainly bases on the idea to reduce the number of objects in the display list since not all objects in the display list created by game engine are necessary for playing the game and scale the complexity of rendering operations. The objective is to maximize the user experience given the communications and computing costs.

\section{E. Other Practical Applications:}

A cloud becomes a useful tool to help mobile users share photos and video clips efficiently and tag their friends in popular social networks as Twitter and
Facebook. MeLog [46] is an MCC application that enables mobile users to share real-time experience (e.g., travel, shopping, and event) over clouds through an automatic blogging. The mobile users (e.g., travelers) are supported by several cloud services such as guiding their trip, showing maps, recording itinerary, and storing images and video. [47] introduces a mobile locationing service allowing users to capture a short video clip about the surrounding buildings. The matching algorithm run on a cloud can use a large amount of information to search for a location of these buildings. Also, One Hour Translation [48] provides an online translation service running on the cloud of AmazonWeb Services. One Hour Translation helps mobile users, especially foreign visitors, receive the information translated in their language through their mobile devices.

A cloud becomes the most effective tool when mobile users require searching services (e.g., searching information, location, images, voices, or video clips).Keyword-based Searching: [49] proposes an intelligent mobile search model using semantic in which searching tasks will be performed on servers in a cloud. This model can analyze the meaning of a word, a phrase, or a complex multi-phase to produce the results quickly and accurately.[50] presents an application using the cloud to perform data searching tasks for mobile users. [50] uses Dessy system[50] to find the users' data, metadata, and context information through desktop search (e.g., indexing, query, and index term stemming, and search relevance ranking) and synchronization techniques.

Voice-based Searching: [52] proposes a search service via a speech recognition in which mobile users just talk to microphone on their devices rather than typing on keypads or touchscreens. [53] introduces the AT\&T speech mashup model that utilizes web services and cloud computing environment to meet the speech service demands of customers. This model optimizes the data transmission in a mobile network, reduces latency, and is flexible in integrating with other services. Several examples are demonstrated (e.g., speak4it, iPizza, and JME local business search).

Tag-based Searching: [54] introduces a photo searching technique based on ontological semantic tags. Mobile users search only recall parameters that are tagged on images before such images are sent to a cloud. The cloud is used for storing and processing images for resource-limited devices.

The current service is designed for the images stored on private cloud computing environment. In the future, it is expected to expand for searching images in a public cloud environment. In addition, there are a mobile-cloud collaborative application [55] to detect traffic lights for the blind, a cloud computing framework [56] to monitor different corners in a house through a mobile device, and some efforts which integrate current services (e.g., BitTorrent, and Mobile Social Network) into the clouds as in [57]. Thereby, we can recognize that MCC is 
probably a prevailing technology trend with numerous applications in the near future.

\section{OPEN RESEARCH ISSUES}

Although some projects of mobile cloud computing have already been deployed around the world, there is still a long way for business implementation, and some research aspects should be considered in further work.

\section{A. Data Delivery:}

Due to the feature of resource-constrains, mobile devices have potential challenges in cloud accessing, consistent accessing, data transmission, and so on. Such challenges can be solved using: special application (service) and middle-ware (provide a platform for all mobile cloud computing systems).

\section{B. Task Division:}

Researchers divide tasks (applications) from mobile devices into multiple sub-tasks and deliver some of them to run in cloud, which is a good solution to the resource limited mobile devices. However, we do not have an optimal strategy or algorithm on how to divide these tasks, which one should be processed by cloud and which one by devices.

\section{Better Service}

The original purpose of mobile cloud computing is providing PC-liked services to mobile terminals. However, as the existing different features between mobile devices and PCs, we cannot directly transplant the services from PCs' platform to mobile devices. Therefore, further research should try to identify the method on how to provide suitable and friendly interactive services for mobile devices.

\section{CONCLUSION}

With the high increasing of data computation in commerce and science, the capacity of data processing has been considered as a strategic resource in many countries. Mobile cloud computing (MCC), as a development and extension of mobile computing (MC) and cloud computing (CC), has inherited the high mobility and scalability, and become a hot research topic in recent years. We conclude that there are three main optimization approaches in $\mathrm{CC}$, which are focusing on the limitations of mobile devices, quality of communication, and division of applications services.

Firstly, using virtualization and image technology can address it effectively, and immigrate task from terminal to loud is also a good way to achieve better results. Secondly, as we know the quality of communication in wired network is better than in wireless network, so reducing the proportion of data delivery in wireless environment is an effective way to improve the quality. In addition, upgrading bandwidth is envisaged to be a simple way to increase performance but it incurs additional cost to users. Deploying an effective elastic application division mechanism is deemed to be the best solution to guarantee the application service in $\mathrm{MCC}$; its complicated, but promising high impact results.

\section{REFERENCES}

[1] S. Perez, Mobile cloud computing: $\$ 9.5$ billion by 2014, http://exoplanet.eu/catalog.php, 2010.

[2] M. Satyanarayanan, Fundamental challenges in mobile computing, in: Proceedings of the Fifteenth Annual ACM Symposium on Principles of Distributed Computing, PODC'96, ACM, New York, NY, USA, 1996, pp. 1-7.

[3] L. Siegele, Let it rise: a special report on corporate it, http://www.economist.com/node /12411882, 2008.

[4] M. Satyanarayanan, Mobile computing, Computer 26 (1993) 81-82.

[5] W. Vogels, A head in the clouds the power of infrastructure as a service, in: Proceedings of the 1st Workshop on Cloud Computing and Applications, CCA'08.

[6]. M. Armbrust, A. Fox, R. Griffith, A. Joseph, R. Katz, A. Konwinski, G. Lee, D. Patterson, A. Rabkin, I. Stoica, Above the clouds: a Berkeley view of cloud computing, Technical Report UCB/EECS-2009-28, 2009.

[7] J. Carolan, S. Gaede, J. Baty, G. Brunette, A. Licht, J. Remmell, L. Tucker, J. Weise, Introduction to cloud computing architecturewhite paper, 2009.

[8] (2009, Sept) Mobile cloud computing subscribers to total nearly one billion by 2014. [Online]. Available: http://www.abiresearch.com/press/ 1484

[9] C. Hewitt, "Orgs for scalable, robust, privacyfriendly client cloud computing," Internet Computing, IEEE, vol. 12, no. 5, pp. 96-99, 2008.

[10] R. Buyya, C. Yeo, and S. Venugopal, "Marketoriented cloud computing: Vision, hype, and reality for delivering it services as computing utilities," in High Performance Computing and Communications, 2008. HPCC'08. 10th IEEE International Conference on. IEEE, 2008, pp. 513.

[11] L. Youseff, M. Butrico, and D. Da Silva, "Toward a unified ontology of cloud computing," in Grid Computing Environments Workshop, 2008. GCE'08. IEEE, 2008, pp. 1-10.

[12] S. Shankar, "Amazon elastic compute cloud," 2009.

[13] A. Zahariev, "Google app engine," Helsinki University of Technology, 2009.

[14] (2011) Microsoft azure homepage. [Online]. 
Available: http://www. windowsazure.com/en-us/

[15] J. McCarthy. (1961) Speech given to celebrate mits centennial. [Online]. Available: http://en.wikipedia.org/wiki/John McCarthy(computerscientist)

[16] (2009) The customer relationship management (crm). [Online]. Available: http://en.wikipedia.org/wiki/Customerrelationshi pmanage ment

[17] B. Rochwerger, D. Breitgand, E. Levy, A. Galis, K. Nagin, I. Llorente, R. Montero, Y. Wolfsthal, E. Elmroth, J. Caceres et ah, "The reservoir model and architecture for open federated cloud computing," IBM Journal of Research and Development, vol. 53, no. 4, pp. 1-11, 2009.

[18] L. Mei, W. Chan, and T. Tse, "A tale of clouds: paradigm comparisons and some thoughts on research issues," in Asia-Pacific Services Computing Conference, 2008. APSCC'08. IEEE. IEEE, 2008, pp. 464-469.

[19] R. Cohen. (2010, O) The cloud computing opportunity by the numbers. [Online]. Available: http://www.elasticvapor.com/2010/05/ cloudcomputing-opportunity- by- numbers. $\mathrm{html}$

[20] M. Ali, "Green Cloud on the Horizon," in Proceedings of the 1st International Conference on Cloud Computing (CloudCom), pp. 451459, December 2009.

[21] http://www.mobilecloudcomputingforum.com/

[22] White Paper, "Mobile Cloud Computing Solution Brief," AEPONA, November 2010.

[23] Jacson H. Christensen, "Using RESTful webservices and cloud computing to create next generation mobile applications," in Proceedings of the 24th ACM SIGPLAN conference companion on Object oriented programming systems languages and applications (OOPSLA), pp. 627-634, October 2009.

[24] L. Liu, R. Moulic, and D. Shea, "Cloud Service Portal for Mobile Device Management," in Proceedings of IEEE 7th International Conference on e-Business Engineering (ICEBE), pp. 474, January 2011.

[25] B. Marrapese. (2010, Dec.) Google ceo: a few years later, the mobile phone becomes a super computer. [Online]. Available: http://www.itnews-blog.com/it/21320.html

[26] S. Chetan, G. Kumar, K. Dinesh, K. Mathew, and M. Abhimanyu, "Cloud computing for mobile world," available at chetan. ueuo. com.

[27] X. Yang, T. Pan, and J. Shen, "On 3G Mobile Ecommerce Platform Based on Cloud Computing," in Proceedings of the 3rd IEEE International Conference on Ubi-Media
Computing (U-Media), pp. 198 - 201, August 2010.

[28] J. Dai, and Q. Zhou, "A PKI-based mechanism for secure and efficient access to outsourced data," in Proceedings of the 2nd International Conference on Networking and Digital Society (ICNDS), vol. 1, pp. 640, June 2010.

[29] Z. Leina, P. Tiejun, and Y. Guoqing, "Research of Mobile Security Solution for Fourth Party Logistics," in Proceedings of the $6^{\text {th }}$ International Conference on Semantics Knowledge and Grid (SKG), pp. 383 - 386, January 2011.

[30] X. Chen, J. Liu*, J. Han, and H. Xu, “ Primary Exploration of Mobile Learning Mode under a Cloud Computing Environment," in Proceedings of the International Conference on E-Health Networking, Digital Ecosystems and Technologies (EDT), vol. 2, pp. 484 - 487, June 2010.

[31] H. Gao and Y. Zhai, "System Design of Cloud Computing Based on Mobile Learning," in Proceedings of the 3rd International Symposium on Knowledge Acquisition and Modeling (KAM), pp. 293 - 242, November 2010.

[32] Jian Li, "Study on the Development of Mobile Learning Promoted by Cloud Computing," in Proceedings of the 2nd International Conference on Information Engineering and Computer Science (ICIECS), pp. 1, December 2010.

[33] W. Zhao, Y. Sun, and L. Dai, "Improving computer basis teaching through mobile communication and cloud computing technology," in Proceedings of the 3rd International Conference on Advanced Computer Theory and Engineering (ICACTE), vol. 1, pp. 452 - 454, September 2010

[34] C. Yin, B. David, and R. Chalon, "Use your mobile computing devices to learn - Contextual mobile learning system design and case studies," in Proceedings of the 2nd IEEE International Conference on Computer Science and Information Technology (ICCSIT), pp. 440, September 2009.

[35] R. Rieger and G. Gay, "Using mobile computing to enhance field study," in Proceedings of the 2nd international conference on Computer support for collaborative learning (CSCL), pp. 218-226, December 1997. Accepted in Wireless Communications and Mobile Computing - Wiley http://onlinelibrary.wiley.com/doi/10.1002/wcm. 1203/abstr act34

[36] R. Ferzli and I. Khalife, "Mobile cloud computing educational tool for image/video processing algorithms," in Digital Signal Processing Workshop and IEEE Signal Processing Education Workshop (DSP/SPE), pp. 
529, March 2011.

[37] L. T. Kohn, J. M. Corrigan, and S. Donaldson, "To Err Is Human: Building a Safer Health System," NATIONAL ACADEMY PRESS Washington, 1999.

[38] D. Kopec, M. H. Kabir, D. Reinharth, O. Rothschild, and J. A. Castiglione, "Human Errors in Medical Practice: Systematic Classification and Reduction with Automated

Information Systems," Journal of Medical Systems, vol. 27, no. 4, pp. 297 - 313, August 2003.

[39] U. Varshney, "Pervasive healthcare and wireless health monitoring," Journal on Mobile Networks and Applications, vol. 12, no. 2-3, pp. 113 - 127, March 2007.

[40] C. Doukas, T. Pliakas, and I. Maglogiannis, “ Mobile Healthcare Information Management unitizing Cloud Computing and Android OS," in Annual International Conference of the IEEE on Engineering in Medicine and Biology Society (EMBC), pp. 1037 - 1040, October 2010.

[41] W-T. Tang, C-M. Hu, and C-Y. Hsu, "A mobile phone based homecare management system on the cloud," in Proceedings of the $3^{\text {rd }}$ International Conference on Biomedical and Informatics (BMEI), vol. 6, pp. 2442, November 2010.

[42] D. B. Hoang, and L. Chen, "Mobile Cloud for Assistive Healthcare (MoCAsH)," in Proceedings of the 2010 IEEE Asia-Pacific Services Computing Conference (APSCC), pp. 325, February 2011.

[43] M. T. Nkosi and F. Mekuria, "Cloud Computing for Enhanced Mobile Health Applications," in Proceedings of the 2nd IEEE International Conference on Cloud Computing Technology and Science, pp. 629, February 2011.

[44] Z. Li, C. Wang, and R. Xu, "Computation floading of to save energy on handheld devices: a partition scheme," in Proceedings of the 2001 international conference on Compilers, architecture, and synthesis for embedded systems (CASES), pp. 238 - 246, November 2001.

[45] S. Wang and S. Dey, "Rendering Adaptation to Address Communication and Computation Constraints in Cloud Mobile Gaming," in IEEE Global Telecommunications Conference (GLOBECOM), pp. 1-6, January 2011.

[46] H. Li and X-S. Hua, "Melog: mobile experience sharing through automatic multimedia blogging," in Proceedings of the 2010 ACM multimedia workshop on Mobile cloud media computing (MCMC), pp. 19-24, 2010

[47] Z. Ye, X. Chen, and Z. Li, "Video based mobile location search with large set of SIFT points in cloud," in Proceedings of the 2010 ACM multimedia workshop on Mobile cloud media computing (MCMC), pp. 25-30, 2010.

[48] http://www.onehourtranslation.com/

[49] V. S. Pendyala and J. Holliday, "Performing Intelligent Mobile Searches in the Cloud Using Semantic Technologies," in IEEE International Conference on Granular Computing, pp. 381, September 2010 .

[50] E. Lagerspetz and S. Tarkoma, "Cloud-assisted mobile desktop search," in Proceedings of the 8th IEEE International Conference on Pervasive Computing and Communications Workshops (PERCOM), pp. 826, May 2010.

[51] E. Lagerspetz, T. Lindholm, and S. Tarkoma, "Dessy: Towards Flexible Mobile Desktop Search," in Proceedings of the DIALM-POMC International Workshop on Foundations of Mobile Computing, August 2007.

[52] G. D. Fabbrizio, T. Okken, and J. G. Wilpon, “A speech mashup framework for multimodal mobile services," in Proceedings of the 2009 international conference on Multimodal interfaces (ICMI-MLMI), pp. 71-78, November 2009.

[53] Cai-Dong Gu, Kan Lu, Jian-Ping Wu, Ying-li Fu, Jing-xiang Li, Chang-shui Xiao, Mao-xin Si, and Zhao-bin Liu, "The Investigation of CloudComputing-based Image Mining Mechanism in Mobile Communication WEB on Android," in Proceedings of the 9th International Conference on Grid and Cooperative Computing (GCC), pp. 408, January 2011.

[54] P. Angin, B. Bhargava, and S. Helal, "A MobileCloud Collaborative Traffic Lights Detector for Blind Navigation," in Proceedings of the 2010 Eleventh International Conference on Mobile Data Management (MDM), pp. 396-401, 2010.

[55] Y-C. Li, I-J. Liao, H-P. Cheng, and W-T. Lee, “A cloud computing framework of free view point real-time monitor system working Accepted in Wireless Communications and Mobile Computing - - Wiley http://onlinelibrary.wiley.com/doi/10.1002/wcm.1 203/abstr act35 on mobile devices," in International Symposium on Intelligent Signal Processing and Communication Systems (ISPACS), pp. 1, January 2011.

[56] W. Zhenyu, Z. Chunhong, J. Yang, and W. Hao, "Towards Cloud and Terminal Collaborative Mobile Social Network Service," in Proceedings of the 2nd IEEE International Conference on Social Computing (SocialCom), pp. 623, September 2010.

[57] I. Kelenyi and J. K. Nurminen, "CloudTorrent - 
Energy-Efficient BitTorrent Content Sharing for Mobile Devices via Cloud Services," in Proceedings of the 7th IEEE on Consumer
Communications and Networking Conference (CCNC), pp. 1, February 2010. 